\title{
A note on the geometry of CHL heterotic strings
}

\author{
Wolfgang Lerche ${ }^{\mathrm{a}}$, Christoph Schweigert ${ }^{\mathrm{a}}$, Ruben Minasian ${ }^{\mathrm{b}}$, Stefan Theisen ${ }^{\mathrm{c}}$ \\ a CERN, CH-1211 Genève 23, Switzerland \\ ${ }^{\mathrm{b}}$ Department of Physics, Yale University, New Haven, CT 06520, USA \\ c Sektion Physik, Universität München, Theresienstraße 37, D-80333 München, Germany
}

Received 19 January 1998

Editor: L. Alvarez-Gaumé

\begin{abstract}
We present a few remarks on disconnected components of the moduli space of heterotic string compactifications on $T_{2}$. We show in particular how the eight dimensional CHL heterotic string can be understood in terms of topologically non-trivial $E_{8} \times E_{8}$ and $\operatorname{Spin}(32) / Z_{2}$ vector bundles over the torus, and that the respective moduli spaces coincide. (C) 1998 Elsevier Science B.V.
\end{abstract}

\section{Introduction}

The recent developments in string theory have made clear that moduli spaces of string compactifications are often connected. This is particularly pronounced for theories with extended supersymmetry. However, even for theories with extended supersymmetry it is in general not true that all string compactifications to the same number of dimensions and with the same amount of supersymmetry are continuously connected. It is indeed well-known that there are disconnected components of theories with $N=2$ supersymmetry in eight and six dimensions, or $N=4$ supersymmetry in four dimensions [1-3]. It would certainly be interesting to have a deeper geometrical understanding of the appearance of such disconnected components, which typically lead to non-simply laced gauge groups.

For compactifications of the heterotic string, disconnected components arise because such compactifications require the choice of a manifold $X$ together with a holomorphic vector bundle on $X$ with structure group, given by either $G=E_{8} \times E_{8}$ or $\operatorname{Spin}(32) / Z_{2}$. The moduli space of holomorphic vector bundles on $X$ can in general have several disconnected components. This applies in particular to the two gauge groups just mentioned when $X$ is a torus. Indeed, for $T_{2}$ compactifications of the heterotic string to $D=8$ there are two known components: the usual component, $\mathscr{M}_{18,2}$, corresponds to the standard Narain moduli space based on the lattice $\Gamma_{18,2}$, while the other component $\mathscr{M}_{10,2}$ corresponds to the CHL string whose moduli space is based on $\Gamma_{10,2}$ [1,2]. Moduli spaces of flat $G$-valued connections over tori had also played a rôle in the recent work of Friedman, Morgan and Witten [4]. Quite generally, disconnected components of moduli spaces correspond to topologically non-trivial 
$G$-bundles. ${ }^{1}$ Fortunately, a rather explicit description of all such disconnected components is known from conformal field theory[8]. This will be reviewed and further discussed in Section 2 of the present letter.

Subsequently, in Section 3 we will then use this description to gain geometrical insight into the nature of the CHL string in eight dimensions. In particular, we will explicitly show that the disconnected components of the $E_{8} \times E_{8}$ and of the $\operatorname{Spin}(32) / Z_{2}$ compactification are equivalent, ie., that they form the same moduli space $\mathscr{M}_{10,2}$. This generalizes the well-known result [5] in the trivial component of the moduli space, namely that ordinary toroidal compactifications of the two heterotic strings map into the same moduli space $\mathscr{M}_{18,2}$.

\section{Flat bundles with non-simply connected structure groups}

We start our discussion of disconnected components of the moduli space with an explicit description of the moduli spaces of flat connections over an elliptic curve $\Sigma$. We fix a compact, real, connected Lie group $G$ which, however, is not necessarily simply connected. Its universal covering group will be denoted by $\tilde{G}$; if $Z:=\pi_{1}(G)$ is the fundamental group of $G$, then $G$ can be obtained from $\tilde{G}$ by dividing out a subgroup of the center of $\tilde{G}$ that is isomorphic to $Z$ :

$$
G=\tilde{G} / Z \text {. }
$$

Let us fix a canonical basis $c_{a}, c_{b}$ of one-cycles on $\Sigma$. Flat connections can be characterized by their holonomies $g_{a}$ and $g_{b}$ around $c_{a}$ and $c_{b}$ which are elements in the universal covering group $\tilde{G}$. After projection to $G$ these elements have to reproduce the single non-trivial relation in the fundamental group of $\Sigma$ :

$$
\pi\left(g_{a} g_{b}\left(g_{a}\right)^{-1}\left(g_{b}\right)^{-1}\right)=\mathrm{e}
$$

which means in $\tilde{G}$ that

$$
g_{a} g_{b}\left(g_{a}\right)^{-1}\left(g_{b}\right)^{-1}=\omega \text { with } \omega \in Z .
$$

As a consequence, the moduli space $\mathscr{M}_{G}$ of flat $G$-connections over $\Sigma$ decomposes into $|Z|$ disconnected components, which are labelled by elements of $Z$ :

$$
\mathscr{M}_{G}=\dot{\cup} \mathscr{M}_{G}^{\omega} \text {. }
$$

It has been shown in [8] that the components with $\omega \neq \mathrm{e}-$ which, as explained above, we call topologically non-trivial components - are isomorphic (as varieties, and up to a rescaling also as complex spaces) to the topologically trivial component of the moduli space over the same elliptic curve, but with another structure group $G^{\omega}$. In other words: we have a fully non-perturbative identity which allows us to trade topological non-triviality for a change in the gauge group.

The change in the structure group corresponds to folding the affine Dynkin diagram ${ }^{2}$ by the automorphism associated to $\omega \in Z$. The relevant folding data of all the groups are listed in Table 1.

Two explicit examples are shown in Fig. 1: in the left column it is shown that the two non-trivial elements in the center of $E_{6}$ give the moduli space of flat $G_{2}$ connections. The total moduli space of topologically trivial and non-trivial $E_{6}$ connections thus has the following three components:

$$
\mathscr{M}_{E_{6}}=\cup_{i=-1,0,1} \mathscr{M}_{E_{6}}^{i} \cong \mathscr{M}_{E_{6}}^{0} \cup \mathscr{M}_{G_{2}}^{0} \cup \mathscr{M}_{G_{2}}^{0} \text {. }
$$

Two comments are in order: we first remark that the case of $A_{n}$ corresponds to vector bundles of rank $n+1$. It seems that to algebraic geometers the following closely related isomorphism has been known: the moduli space $\mathscr{M}(r, d)$ of vector bundles of rank $r$ and degree $d$ over an elliptic curve is isomorphic to the moduli space

\footnotetext{
${ }^{1}$ As usual in physics, we call these bundles, topologically non-trivial, because they cannot be deformed as flat bundles to the trivial bundle. In the case of a two-dimensional torus such bundles are even non-trivial as topological bundles.

${ }^{2}$ This is related to but different as Ref. [4], where automorphisms of non-affine Dynkin diagrams were considered.
} 
Table 1

\begin{tabular}{cccc}
\hline$G$ & $\omega$ & $N$ & $G^{\omega}$ \\
\hline$A_{n}$ & $\left(\sigma_{n+1}\right)^{(n+1) / N}$ & $N<n+1$ & $A_{((n+1) / N)-1}$ \\
$A_{n}$ & $\sigma_{n+1}$ & $n+1$ & $\{0\}$ \\
$B_{n}$ & $\sigma_{v}$ & 2 & $\tilde{B}_{n-1}^{(2)}$ \\
$C_{2 n}$ & $\sigma$ & 2 & $\tilde{B}_{n}^{(2)}$ \\
$C_{2 n+1}$ & $\sigma$ & 2 & $C_{n}$ \\
$D_{n}$ & $\sigma_{v}$ & $C_{n-2}$ & $B_{n}$ \\
$D_{2 n}$ & $\sigma_{s}$ & 2 & $C_{n-1}$ \\
$D_{2 n+1}$ & $\sigma_{s}$ & 4 & $G_{2}$ \\
$E_{6}$ & $\sigma$ & 3 & $F_{4}$ \\
$E_{7}$ & $\sigma$ & 2 & 2 \\
\hline
\end{tabular}

$\mathscr{M}(x(r, d), 0)$, where $x(r, d)$ denotes the greatest common divisor of $r$ and $d$. Also notice (for $\left.A_{n}\right)$ that if $\omega$ generates the whole center, then the moduli space is just a single point. Our second comment is about the symbol $\tilde{B}_{n}^{(2)}$ (c.f., right part of Fig. 1): this denotes the only twisted affine Lie algebra which has characters that span a unitary representation of the modular group. In this case, the moduli space can be build from the maximal torus of this algebra and its affine Weyl group precisely in the same way as the other moduli spaces can be built from the data of the untwisted affine Lie algebras.

More specifically, any connection in the topologically trivial sector can be described by two commuting elements $g_{a}, g_{b}$ which are only determined up to a simultaneous conjugation with an element of $G$; hence we can assume without loss of generality that they are both elements of a fixed maximal torus of $G$. We write

$$
g_{a}=\exp \left(2 \pi \mathrm{i} h_{a}\right) \quad \text { and } \quad g_{b}=\exp \left(2 \pi \mathrm{i} h_{b}\right) .
$$

The remaining gauge transformations are taken into account by the diagonal action of the Weyl group $W$ on both maximal tori, so that the moduli space is

$$
\mathscr{M}_{G}=\left(\boldsymbol{g}_{0} / L \oplus \boldsymbol{g}_{0} / L\right) / W
$$

where $\boldsymbol{g}_{0}$ is the Cartan subalgebra of the Lie algebra of $G$, and $L$ is the coroot-lattice so that $\boldsymbol{g}_{0} / L$ is just the maximal torus. In the case of $\tilde{B}_{r}^{(2)}, g_{0}$ is an abelian Lie algebra of rank $r, L$ is the root lattice (not the coroot lattice) of $B_{r}$, and $W$ is the Weyl group of $\operatorname{Spin}(2 r+1)$ or, equivalently, of $\operatorname{Sp}(2 r)$. It was shown in [8] that the disconnected components of the moduli space can be described using the analogous data of the group $G^{\omega}$.

Let us make this explicit for the case of Spin(32), which is relevant for the eight dimensional heterotic string compactifications to be discussed later. Usually one describes the Lie algebra of Spin(32) by antisymmetric
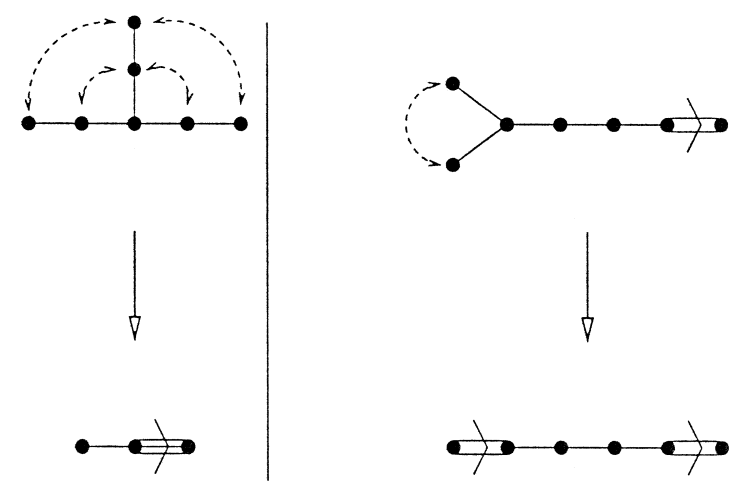

Fig. 1. Some foldings of affine Dynkin diagrams. 
matrices; here we prefer to work in a basis where the Cartan subalgebra is realized by diagonal matrices. This is achieved by the introduction of complex fermions $\left(\psi_{i}, \bar{\psi}_{i}\right), i=1 \ldots 16$. In this basis, the boundary conditions for a world sheet of genus 1 and modular parameter $\tau$ and for winding numbers $L_{1}$ and $L_{2}$ in the bosonic sector are:

$$
\psi_{i}(z+n+m \tau)=\exp \left(2 \pi \mathrm{i}\left(n h_{a}^{i} L_{1}+m h_{b}^{i} L_{2}\right)\right) \psi_{i}(z),
$$

The corresponding partition function can be computed explicitly [7].

We now extend this calculation to the topologically non-trivial bundles. They are again described by the monodromies $g_{a}$ and $g_{b}$ along the non-trivial cycles of the torus. Here $g_{a}$ and $g_{b}$ are group elements in Spin(32) which obey the condition

$$
g_{a} g_{b} g_{a}^{-1} g_{b}^{-1}=\omega,
$$

where $\omega$ is the element in the center of Spin(32) that corresponds to the spinor conjugacy class. Notice that in contrast to the topologically trivial sector, the monodromies now do not commute any more, and the relations of the fundamental group $\pi_{1}\left(T^{2}\right) \cong \boldsymbol{Z} \times \boldsymbol{Z}$ are only reproduced after projection to $\operatorname{Spin}(32) / \boldsymbol{Z}_{2}$. According to the automorphism presented above, the moduli space $\mathscr{M}_{1}$ of the topologically non-trivial $\operatorname{Spin}(32) / \boldsymbol{Z}_{2}$ bundle is isomorphic to the moduli space of topologically trivial Spin(17)-bundles. Hence its dimension is $\operatorname{dim}_{C} \mathscr{M}_{1}=$ $\operatorname{rank}(\operatorname{Spin}(17))=8$.

More generally, a simultaneous conjugation of $g_{a}$ and $g_{b}$ with an element in $\operatorname{Spin}(2 n)$ can be used to bring the general solution of (2.9) to the form

$$
g_{a}=\exp \left(2 \pi \mathrm{i}\left(h_{0}+h_{a}\right) \quad g_{b}=A \exp \left(2 \pi \mathrm{i} h_{b}\right)\right.
$$

where $h_{0}, h_{a}$ and $h_{b}$ are elements in the Cartan subalgebra of $\operatorname{spin}(2 n)$ and $A$ is a group element which upon conjugation reproduces on the Cartan subalgebra the action of the following Weyl transformation:

$$
\begin{aligned}
& w\left(\alpha^{(i)}\right)=\alpha^{(n-i)} \quad \text { for } \quad i=1 \ldots n-1 \\
& w\left(\alpha^{(n)}\right)=-\theta=-\alpha^{(1)}-\alpha^{(n-1)}-\alpha^{(n)}-2 \sum_{i=2}^{n-2} \alpha^{(i)} .
\end{aligned}
$$

$h_{0}$ is a specific element of the Cartan subalgebra which is described in more detail in [8]. Moreover, the elements $h_{a}$ and $h_{b}$ are restricted by the condition

$$
A h_{a} A^{-1}=h_{a} \quad \text { and } \quad A h_{b} A^{-1}=h_{b} .
$$

This condition implies that only half of the possible Wilson lines in the topologically trivial sector survives. This accounts for the reduction of the dimension of the moduli space.

An explicit description of the possible Wilson lines $h_{a}$ and $h_{b}$ is as follows (for notation, details and a derivation see the appendix): in a suitable basis the general element of the Cartan subalgebra can be written as

$$
\sum_{i=1}^{n} \lambda_{i}\left(\mathscr{E}_{i, i}-\mathscr{E}_{i+n, i+n}\right)
$$

where $\mathscr{E}_{i, j}$ is the $2 n \times 2 n$ matrix with only zero entries, except one. The subalgebra of the Cartan subalgebra that is invariant under conjugation with $A$ is given by those elements for which

$$
\lambda_{i}=\lambda_{n+1-i}
$$

it is an $n / 2$-dimensional subalgebra. 


\section{Geometric realization of the $8 \mathrm{~d}$ CHL compactification}

We now come to compactifications of the heterotic string to 8 dimensions. The compactification space has to be a Calabi-Yau manifold, and hence be the two-dimensional torus. Moreover, one has to choose a gauge bundle over this compactification manifold. The conditions for anomaly cancellation require the structure group of this bundle to be either $G_{1}=E_{8} \times E_{8}$ or $G_{2}=\operatorname{Spin}(32) / Z_{2}$. Since the Calabi-Yau metric on the torus is flat, anomaly cancellation tells us that the background gauge field on the bundle has to be flat as well.

Let us consider first the case of $G_{2}=\operatorname{Spin}(32) / Z_{2}$. This group is not simply connected, and we have seen that in this case the moduli space $\mathscr{M}$ of flat connections has more than one connected component. More precisely, one finds that $\pi_{0}(\mathscr{M})=\pi_{1}\left(G_{2}\right)=Z_{2}$. A similar logic applies to $G_{1}=E_{8} \times E_{8}$; in this case the outer automorphism is just the permutation of the two $E_{8}$ factors, and again we have two connected components of the moduli space.

We will now show that the component $\mathscr{M}_{1}$ gives the CHL compactification of the heterotic string. This affords in particular a geometric description of the CHL compactification.

To fix the Wilson lines, we choose $n / 2$ elements $\theta_{i} \in I R \bmod \boldsymbol{Z}$ and $n / 2$ elements $\phi_{i} \in I R \bmod \boldsymbol{Z}$. Given a world sheet of genus 1 and modular parameter $\tau$ and in the sector with winding numbers $L_{1}$ and $L_{2}$ in the bosonic sector, the boundary conditions for the $2 n$ complex fermions are in the direction of the $a$-cycle

$$
\psi^{i}(z+1)=R\left(\mathrm{e}^{2 \pi \mathrm{i} L_{1}\left(h_{a}+h_{0}\right)}\right)^{i i} \psi^{i}(z)= \begin{cases}(\mathrm{i}){ }^{L_{1}} \mathrm{e}^{2 \pi \mathrm{i} \theta_{i} L_{1}} \psi^{i}(z) & \text { for } i=1 \ldots n / 2 \\ (\mathrm{i}){ }^{L_{1}} \mathrm{e}^{2 \pi \mathrm{i} \theta_{n+1-i} L_{1}} \psi^{i}(z) & \text { for } i=n / 2+1 \ldots n \\ (-\mathrm{i})^{L_{1}} \mathrm{e}^{-2 \pi \mathrm{i} \theta_{i-n} L_{1}} \psi^{i}(z) & \text { for } i=n+1 \ldots 3 / 2 n \\ (-\mathrm{i})^{L_{1}} \mathrm{e}^{-2 \pi \mathrm{i} \theta_{2 n+1-i} L_{1}} \psi^{i}(z) & \text { for } i=3 / 2 n+1 \ldots 2 n\end{cases}
$$

and in the direction of the $b$-cycle for odd winding $L_{2}$

$$
\begin{aligned}
\psi^{i}(z+\tau)= & \sum_{j=1}^{2 n} R\left(A^{L_{2}} \mathrm{e}^{2 \pi \mathrm{i} h_{b} L_{2}}\right)^{i j} \psi_{j}(z) \\
= & \begin{cases}\mathrm{ie}^{2 \pi \mathrm{i} \phi_{i} L_{2}} \psi^{2 n+1-i}(z) & \text { for } i=1 \ldots n / 2 \\
\mathrm{ie}^{2 \pi \mathrm{i} \phi_{n+1-i} L_{2}} \psi^{2 n+1-i}(z) & \text { for } i=n / 2+1 \ldots n \\
-\mathrm{ie}^{-2 \pi \mathrm{i} \phi_{i-n} L_{2}} \psi^{2 n+1-i}(z) & \text { for } i=n+1 \ldots 3 / 2 n \\
-\mathrm{ie}^{-2 \pi \mathrm{i} \phi_{2 n+1-i} L_{2}} \psi^{2 n+1-i}(z) & \text { for } i=3 / 2 n+1 \ldots 2 n\end{cases}
\end{aligned}
$$

and similarly for even winding. We see that the fermions come in groups of four, and the boundary conditions of such a group of four fermions is described by two parameters $\theta_{i}$ and $\phi_{i}$. For $\operatorname{Spin}(32) / Z_{2}$ we have the following groups of indices: $(1,16,8,9),(2,15,7,10),(3,14,6,11),(4,13,5,12)$.

This should be compared to the topologically non-trivial component of the moduli space of the $E_{8} \times E_{8}$ string. Here, we impose the following boundary conditions: along one cycle, say $c_{a}$, we introduce an ordinary Wilson line, while the monodromy along the other cycle $c_{b}$ interchanges the two $E_{8}$ factors and hence in particular the fermionic operators that are used to make up their Cartan subalgebras. This leads to an exchange of two complex fermions, which in turn can be described in terms of real fermions. Up to different phases (which can be compensated by the Wilson lines and a relabeling of the fermions), we recover exactly the same behaviour as for the non-trivial component of the $\operatorname{Spin}(32) / Z_{2}$ string.

We have thus shown that the CHL string has a natural geometric description and that the equivalence of heterotic compactifications based on $E_{8} \times E_{8}$ on the one hand and on $\operatorname{Spin}(32) / Z_{2}$ on the other hand holds in the topologically non-trivial component of the moduli space of connections as well. 
Notice that so far we have been concerned only with the moduli space $\mathscr{M}_{G}$ of flat connections, i.e. the moduli space of the Wilson lines. The full moduli space of heterotic compactifications is locally a product of this moduli space and the moduli space $\mathscr{M}_{2,2}$ of 2-tori:

$$
\mathscr{M}_{18,2} \sim \mathscr{M}_{\mathrm{Spin}(32)} \times \mathscr{M}_{2,2} \sim \mathscr{M}_{E_{8} \times E_{8}} \times \mathscr{M}_{2,2}, \quad \mathscr{M}_{10,2} \sim \sim \mathscr{M}_{\mathrm{Spin}(17)} \times \mathscr{M}_{2,2} / \boldsymbol{Z}_{2} .
$$

In this context, the following observation seems to be intriguing: for the Narain compactification, the moduli space is described by either $E_{8} \times E_{8}$ or Spin(32) bundles, and both groups appear as possible gauge enhancements (by allowing general rotations involving $\Gamma_{2,2}$, one can extend Spin(32) to Spin(36)). In the CHL compactification, the Wilson lines are described by Spin(17) which is not simply-laced and does not appear as a possible gauge group of the CHL-string. However, its dual (obtained by reversing the arrow in the [non-extended] Dynkin diagram), which is $\mathrm{Sp}(16)$, does (by allowing general rotations, this can similarly be extended to $S p(20)$ ). In other words, and this seems to be a general rule, the gauge group that one canonically obtains is not given by the structure group $G$ of the bundle, but by its dual, $G^{\vee}$.

A similar structure, however related to affine Dynkin diagrams, was found in Ref. [4]. In fact, this pattern is familiar from many contexts, like for example in $N=2$ gauge theories [9].

Given that the CHL string has this geometric interpretation which puts it on the same footing as the usual Narain compactification, it is natural to ask what its $F$-theory dual is; this will be addressed in a future publication.

\section{Acknowledgements}

We would like to thank Peter Mayr for collaborating on $F$-theory related aspects of this work.

\section{Appendix A. Conjugations in $\operatorname{Spin}(2 n)$}

We need the group elements $g_{a}$ and $g_{b}$ that describe the monodromies around $c_{a}$ and $c_{b}$ explicitly in the $2 n$-dimensional representation of $\operatorname{Spin}(2 n)$, the vector representation. Notice that this is not a representation of $\operatorname{Spin}(2 n) / \boldsymbol{Z}_{2}$, and as a consequence, the element $\omega$ in the center is not represented by the identity.

To this end we first give the generators of the Lie algebra $s o(2 n)$ in the $2 n$-dimensional representation. Denote by $\mathscr{E}_{i, j}$ the $2 n \times 2 n$ matrix which has only zeros as entries, except for 1 in the $i$-th row and $j$-th column. Again it is convenient not to work with antisymmetric matrices but to perform a unitary transformation with the matrix

$$
U:=\frac{1}{\sqrt{2}} \sum_{i=1}^{n} \mathrm{i} \mathscr{E}_{i, i}-\mathscr{E}_{i+n, i+n}-\mathrm{i} \mathscr{E}_{i, n+i}-\mathscr{E}_{n+i, i}
$$

This corresponds to the usual introduction of complex fermions and allows to represent elements of the Cartan subalgebra by diagonal matrices. The matrices $B$ of the $2 n$-dimensional representation of the Lie group $\operatorname{Spin}(2 n)$ are then characterized by the fact that $B^{t} K B=K$, where $K$ is the matrix

$$
K:=U^{t} U=\sum_{i=1}^{n} \mathscr{E}_{i, i+n}+\mathscr{E}_{i+n, i}
$$


The generators of the Lie algebra $\operatorname{spin}(2 n)$ in a Cartan-Weyl basis are then represented as follows:

$$
\begin{aligned}
& R\left(E_{+}^{i}\right)=\mathscr{E}_{i, i+1}-\mathscr{E}_{i+n+1, i+n} \text { for } i=1 \ldots n-1 \\
& R\left(E_{-}^{i}\right)=\mathscr{E}_{i+1, i}-\mathscr{E}_{i+n, i+n+1} \text { for } i=1 \ldots n-1 \\
& R\left(E_{+}^{n}\right)=\mathscr{E}_{n-1,2 n}-\mathscr{E}_{n, 2 n-1} \\
& R\left(E_{-}^{n}\right)=\mathscr{E}_{2 n, n-1}-\mathscr{E}_{2 n-1, n} \\
& R\left(H^{i}\right)=\mathscr{E}_{i, i}-\mathscr{E}_{i+1, i+1}-\mathscr{E}_{i+n, i+n}+\mathscr{E}_{i+n+1, i+n+1} \quad \text { for } i=1 \ldots n-1 \\
& R\left(H^{n}\right)=\mathscr{E}_{n-1, n-1}+\mathscr{E}_{n, n}-\mathscr{E}_{2 n-1,2 n-1}-\mathscr{E}_{2 n, 2 n} .
\end{aligned}
$$

Finally, we need the generators for the $s u(2)$-subalgebra of $\operatorname{spin}(2 n)$ generated by the highest root $\theta$ of $\operatorname{spin}(2 n)$ :

$$
\begin{aligned}
& R\left(E_{+}^{\theta}\right)=\mathscr{E}_{1, n+2}-\mathscr{E}_{2, n+1} \\
& R\left(E_{-}^{\theta}\right)=\mathscr{E}_{n+2,1}-\mathscr{E}_{n+1,2} \\
& R\left(H^{\theta}\right)=\mathscr{E}_{1,1}+\mathscr{E}_{2,2}-\mathscr{E}_{n+1, n+1}-\mathscr{E}_{n+2, n+2} .
\end{aligned}
$$

We claim that the following matrix is a solution:

$$
A:=\sum_{i=1}^{n}-\mathrm{i} \mathscr{E}_{i, 2 n+1-i}+\mathrm{i} \mathscr{E}_{2 n+1-i, i}
$$

One can check that it has the following properties: $A^{2}=1, A$ is in $S O(2 n)$, which follows from the fact that

$$
U A U^{+}=-\sum_{i=1} 2 n \mathscr{E}_{i, 2 n+1-i}
$$

which is a real orthogonal matrix. Moreover, one has

$$
\begin{aligned}
& A R\left(E_{ \pm}^{i}\right) A=-R\left(E_{ \pm}^{n-i}\right) \text { for } i=1 \ldots n-1 \\
& A R\left(E_{ \pm}^{n}\right) A=R\left(E^{\mp \theta}\right) \\
& A R\left(H^{i}\right) A=R\left(H^{n-i}\right) \text { for } i=1 \ldots n-1 \\
& A R\left(H^{n}\right) A=R\left(H^{\theta}\right) .
\end{aligned}
$$

This reproduces correctly the action of the Weyl group element $w$ described in (2.11). Using the results in [8] one may show that

$$
R\left(h_{0}\right)=\frac{1}{4} \sum_{i=1}^{n}\left(\mathscr{E}_{i, i}-\mathscr{E}_{n+i, n+i}\right)
$$

One readily verifies that this satisfies $e^{2 \pi i h_{0}} A e^{-2 \pi i h_{0}} A^{-1}=\omega$ with $R(\omega)=-\sum_{i=1}^{n}\left(\mathscr{E}_{i, i}+\mathscr{E}_{n+i, n+i}\right)$, as implied by (2.9).

\section{References}

[1] S. Chaudhuri, G. Hockney, J. Lykken, Phys. Rev. Lett. 75 (1995) 2264, hep-th/9505054.

[2] S. Chaudhuri, J. Polchinski, Phys. Rev. D 52 (1995) 7168 , hep-th/9506048.

[3] J. Schwarz, A. Sen, Phys. Lett. B 357 (1995) 323, hep-th/9507027; S. Chaudhuri, D. Lowe, Nucl. Phys. B 459 (1996) 113, hep-th/9508144.

[4] R. Friedman, J. Morgan, E. Witten, Commun. Math. Phys. 187 (1997) 679, hep-th/9701162.

[5] P. Ginsparg, Phys. Rev. D 35 (1987) 648.

[6] K.S. Narain, Phys. Lett. B 169 (1986) 41.

[7] K.S. Narain, M. Sarmadi, E. Witten, Nucl. Phys. B 279 (1987) 369 .

[8] C. Schweigert, Nucl. Phys. B 492 (1997) 743 , hep-th/9611092.

[9] E. Martinec, N. Warner, Nucl. Phys. B 459 (1996) 97, hep-th/9509161. 